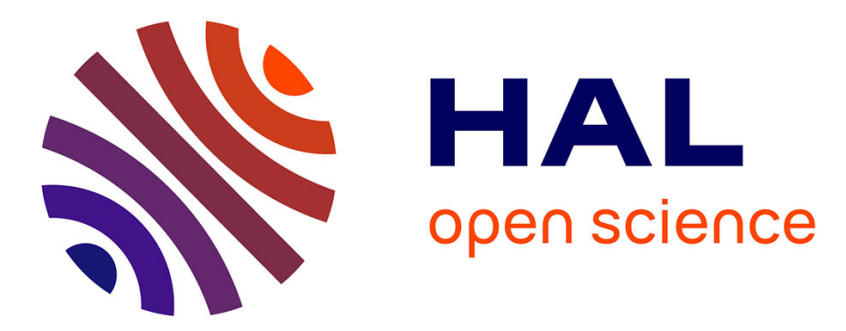

\title{
Mesure de haute résolution du rayonnement gamma issu de la réaction $(\mathrm{n}, \gamma)$ \\ P.H.M. van Assche
}

\section{To cite this version:}

P.H.M. van Assche. Mesure de haute résolution du rayonnement gamma issu de la réaction (n, $\gamma$ ). Revue de Physique Appliquée, 1969, 4 (2), pp.269-270. 10.1051/rphysap:0196900402026900 . jpa00243255

\section{HAL Id: jpa-00243255 https://hal.science/jpa-00243255}

Submitted on 1 Jan 1969

HAL is a multi-disciplinary open access archive for the deposit and dissemination of scientific research documents, whether they are published or not. The documents may come from teaching and research institutions in France or abroad, or from public or private research centers.
L'archive ouverte pluridisciplinaire HAL, est destinée au dépôt et à la diffusion de documents scientifiques de niveau recherche, publiés ou non, émanant des établissements d'enseignement et de recherche français ou étrangers, des laboratoires publics ou privés. 


\title{
MESURE DE HAUTE RÉSOLUTION DU RAYONNEMENT GAMMA ISSU DE LA RÉAGTION $(n, \gamma)$
}

\author{
P. H. M. VAN ASSGHE, \\ S.C.K.-C.E.N., Mol, Belgique.
}

\begin{abstract}
Résumé. - On a comparé les caractéristiques d'un détecteur semiconducteur et un spectromètre à diffraction, utilisés dans l'analyse des gamma de capture de neutrons.

Abstract. - A comparison has been made between a semiconductor detector and a diffraction spectrometer when used in the analysis of neutron capture gamma radiation.
\end{abstract}

Pour aborder l'analyse du rayonnement $\gamma$ issu de la réaction $(\mathrm{n}, \gamma)$, dit " gamma de capture », il existe actuellement deux types de spectromètres : à semiconducteurs et à diffraction. Nous comparerons la résolution, le pouvoir de discrimination, la précision et l'application à l'analyse des gamma de capture.

I. La résolution. - La résolution des détecteurs semiconducteurs est conditionnée par : i) la largeur intrinsèque due aux phénomènes de production de porteurs de charge (facteur de Fano) et ii) la résolution de l'équipement électronique. En ne tenant compte que de la largeur intrinsèque du détecteur, on pourra estimer une limite inférieure des largeurs de pics actuellement accessibles. Des études récentes [1] avec un semiconducteur $\mathrm{Ge}(\mathrm{Li})$ semblent conduire à un facteur de Fano entre 0,12 et 0,13 et aux largeurs intrinsèques indiquées sur la figure 1 .

La résolution d'un spectromètre à diffraction est définie par $\Delta E=\alpha E^{2} \cdot 1 / n$, où $\Delta E$, largeur à mihauteur; $E$, énergie du rayonnement gamma diffracté; $n$, ordre de diffraction. Dans le cas du spectromètre installé au réacteur DR-3 à Risø [2], $\alpha$ est égal à $1,2 \times 10^{-5}$ (avec $E$ en $\mathrm{keV}$ ). Les courbes de $\Delta E$ en fonction de l'énergie $\gamma$ sont également représentées (fig. 1). Il est courant d'observer des transitions $\gamma$ intenses jusqu'au $5^{\mathrm{e}}$ ordre de diffraction.

II. Le pouvoir de discrimination. - Dans les spectres $\gamma$ où le nombre de transitions est élevé, l'obser-

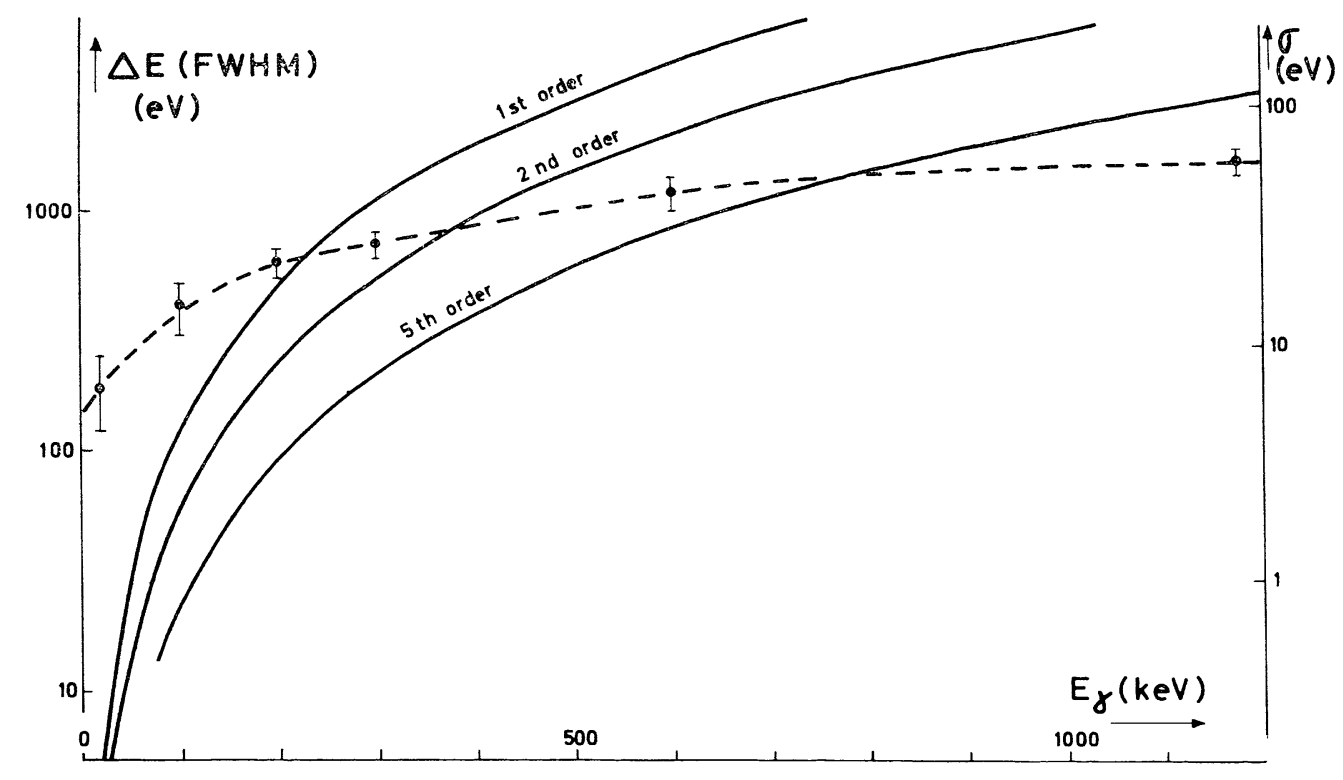

FIG. 1. - Largeur à mi-hauteur $\Delta E$ en fonction de l'énergie $\gamma$. Les courbes pleines correspondent aux différents ordres de diffraction, la courbe pointillée reprend les données de [1]. 
TABLEAU I

$\begin{array}{cccc}E_{\gamma 1}{ }^{a)} & E_{\gamma 2} & \text { SPECTROMÈTRE } & \text { RÉF. } \\ 889,15 \pm 0,130 & 1120,30 \pm 0,220 & - & - \\ 889,60 \pm 0,150 & 1120,65 \pm 0,120 & \text { diffr. } & {[3]} \\ 889,15 \pm 0,080 & 1120,52 \pm 0,080 & \text { diffr. } & {[4]} \\ 889,21 \pm 0,100 & 1120,42 \pm 0,100 & \mathrm{Ge}(\mathrm{Li}) & {[6]} \\ 889,25 \pm 0,070 & 1120,50 \pm 0,070 & \mathrm{Ge}(\mathrm{Li}) & {[7]}\end{array}$

a) Énergies $\gamma$ en $\mathrm{keV}$.

vation des transitions à faible intensité en présence de transitions plus intenses s'avère importante. Un spectromètre à diffraction sépare facilement deux transitions, distantes d'un intervalle d'énergie égal à une fois la résolution à cette énergie, et dont les intensités diffèrent d'un facteur $10^{4}$. Notons en plus qu'une énergie $\gamma$ déterminée se manifeste par un pic diffracté bien défini; cette même réponse se répète dans les ordres supérieurs, mais chaque fois avec une résolution améliorée.

III. La précision. - La précision d'une mesure d'énergie à l'aide d'un détecteur semiconducteur dépend de la symétrie des photopics, de la linéarité de conversion et de la calibration en énergie. Les valeurs de l'erreur standard $\sigma$ obtenues actuellement sont de 50 à $100 \mathrm{eV}$. Un spectromètre à diffraction peut fournir des erreurs standard $\sigma$ allant de $1 \mathrm{eV}\left(E_{\gamma} \sim 50 \mathrm{keV}\right)$ à $100 \mathrm{eV}\left(E_{\gamma} \sim 1 \mathrm{MeV}\right)$. Le tableau I contient une liste comparative des transitions $\gamma$ dans ${ }^{46} \mathrm{Ti}$, mesurées avec les deux types de spectromètre.

Une mesure récente [8] de la réaction ${ }^{55} \mathrm{Mn}(\mathrm{n}, \gamma)^{56} \mathrm{Mn}$ nous a fourni, entre autres, trois transitions permettant d'établir la combinaison suivante :

$(26,6043 \pm 0,0004) \mathrm{keV}+(83,8990 \pm 0,0015) \mathrm{keV}$ $-(110,5053 \pm 0,0037) \mathrm{keV}=0,0020 \pm 0,0040 \mathrm{keV}$.

IV. Application à l'analyse des $\gamma$ de capture. Pour la plupart des spectres de $\gamma$ de capture de basse énergie $\left(E_{\gamma}<2 \mathrm{MeV}\right)$, l'analyse dépend d'une façon critique des trois facteurs précédents. Les deux types de spectromètre présentent à peu près la même efficacité totale, celle-ci étant déterminée principalement par les angles solides entre la cible ou la source de neutrons dans le réacteur et le système de détection. En partant de la figure 1, on peut déduire l'énergie $\gamma$ vers laquelle le détecteur semiconducteur devient supérieur au spectromètre à diffraction; elle se situe à environ $800 \mathrm{keV}$. Cette valeur a été confirmée récemment dans l'analyse de la réaction ${ }^{141} \operatorname{Pr}(n, \gamma){ }^{142} \operatorname{Pr}:$ le spectre de basses énergies $\left(E_{\gamma}<1,5 \mathrm{MeV}\right)$ a été étudié par un détecteur semiconducteur avec anneau antiCompton à Los Alamos et par le spectromètre à diffraction de Risø. Du tableau VI de [9], il ressort que le système jonction-anneau anticoïncidence devient supérieur vers 700 à $800 \mathrm{keV}$. Entre-temps, la résolution du spectromètre de Risø s'est améliorée d'un facteur 3, ce qui pourrait déplacer la région de transition au-dessus de $1 \mathrm{MeV}$.

Il faut noter enfin que l'établissement d'un schéma de niveaux est tributaire du nombre de combinaisons fortuites [10-12]. Dans le cas de ${ }^{56} \mathrm{Mn}$, où l'on observe environ 30 transitions $\gamma$ entre 26 et $505 \mathrm{keV}$, ce nombre est inférieur à 2 ou 3 . Le même spectre mesuré avec un détecteur semiconducteur pourrait donner lieu à plus de 20 combinaisons fortuites. Cependant, le schéma construit à partir des mesures par diffraction ne contient que 26 combinaisons d'énergie en tout. Ceci prouve qu'avec la précision obtenue actuellement avec les détecteurs semiconducteurs, on n'arrive pas à établir ce schéma de niveaux; pour obtenir ceci, il faudra compléter ces mesures par d'autres données, par exemple des mesures de coïncidence $\mathrm{Ge}(\mathrm{Li})-\mathrm{Ge}(\mathrm{Li})$. Il se peut qu'un tel système arrive à établir un schéma de niveaux avec la même certitude que celle fournie par un spectromètre à diffraction, bien que les premiers résultats ne soient pas concluants [13].

\section{BIBLIOGRAPHIE}

[1] Palms (J. M.) et al., Nucl. Instr. Methods, 1968, 64, 310.

[2] Schult (O. W. B.) et al., Intern. Conf. Nucl. Phys. with Reactor Neutrons, A.N.L. 6797, 1963, p. 111, et ID., Kerntechnik, 1963, 5, 17 et 19.

[3] Reidy (J. J.) et Wiedenbeck (M. L.), Nucl. Phys., 1965, 70, 518.

[4] Parsignault (D.), Phys. Letters, 1965, 18, 36.

[5] VAN Assche (P.) et al., Nucl. Phys., 1966, 84, 661.
[6] Ramayya (A. V.) et al., Phys. Letters, 1967, 24 B, 49.

[7] Black (W. W.) et HEATH (R. L.), Nucl. Phys., 1967, A 90, 650 .

[8] VAN ASsche (P. H. M.) et al., à paraître.

[9] Kern (J.) et al., Phys. Rev., 1968, 173, 1133.

[10] Schult (O. W. B.), Z. Naturforsch., 1961, 16 A, 927.

[11] SMirnov (A. I.) et al., Nucl. Instr. Methods, 1968, 60, 103.

[12] Schult (O. W. B.), BNL-10996, 1967.

[13] Bolotin (H. H.), Phys. Rev., 1968, 168, 1317. 